\title{
Clinical Spectrum of COVID-19 in a Mexican Pediatric Population
}

\author{
Edgar Bustos-Cordova, Daniela Castillo-Garcia, Magdalena Ceron-Rodriguez and Nadia Soler- \\ QUINONES \\ From Emergency Department, Hospital Infantil de México Federico Gómez, Mexico.
}

Correspondence to: Dr Daniela Castillo-García, 162 Doctor Marquez Street, Col Doctores, Mexico City, Postal Code 06400, dracastillogarcia@gmail.com Received: September 14, 2020; Initial review: October 08, 2020, Accepted: December 14, 2020.

\begin{abstract}
Objective: To describe the broader clinical spectrum of COVID-19 in children. Methods: In this descriptive, prospective study, we included confirmed pediatric patients with COVID-19 who presented to the emergency department of a pediatric tertiary care center from April to July, 2020. All patients were confirmed by the SARS-CoV-2 RT-PCR test, and we analyzed 24 symptoms and 25 signs. Results: Among the 50 patients with COVID-19, the most common symptoms were fever, excessive cry and dry cough; digestive symptoms were frequently found $(24 \%)$. The most common signs were pharyngeal erythema and irritability. Conclusion: Clinicians should recognize that the clinical spectrum of COVID-19 in children is wider than previously described, often with nonspecific signs and symptoms, and digestive symptoms should raise suspicion.
\end{abstract}

Keywords: Diagnosis, Gastrointestinal symptoms, Presentation, SARS-CoV-2.
$\mathrm{T}$ he incidence of coronavirus disease (COVID19) in Mexico began to escalate rapidly in April, 2020. By August 7, 12,052 cases of COVID-19 in children were confirmed in Mexico, with 188 deaths [1]. From the beginning of the COVID-19 pandemic, it has become evident that the spectrum of manifestations in children is different from those seen in adults. However, most of the clinical descriptions have been made from retrospective studies addressing a narrow number of manifestations. A metaanalysis [2] and a systematic review [3] evaluated fewer than ten signs and symptoms. The aim of this study was to describe a broader clinical spectrum of COVID-19 in children.

\section{METHODS}

We conducted a prospective study in the emergency department of a pediatric tertiary care center from April to July, 2020. We included patients $<18$ years of age, with a history in the week before inclusion of at least one of the following criteria: a) one respiratory symptom, b) one gastrointestinal symptom or c) fever and recent exposure to a confirmed COVID-19 case. Patients with tracheostomy, severe neurologic underlying conditions, use of sedatives in the last week or children not accompanied by the primary caretaker were excluded. All cases were tested with severe acute respiratory syndrome coronavirus 2 (SARS-CoV-2) reverse transcriptase-polymerase chain reaction (RT-PCR) nasopharyngeal swabs. For the patients who met the inclusion criteria, we looked for 24 symptoms present prior to evaluation at the emergency department and 25 signs at the physical exploration, and the information was gathered by attending physicians. Gastrointestinal symptoms were defined as the presence of diarrhea, vomiting, nausea or abdominal pain. We defined pneumonia as the presence of one of the following: increased work of breathing or oximetry $<93 \%$.

We analyzed the data from the COVID-19 confirmed patients; the information was analyzed using case counting and descriptive statistics, and calculating median (range), quartiles and percentages. To describe the chronology of the appearance of symptoms, we performed diagrams for each patient. A horizontal line represents the time (days) before admission. Time zero represents the day of onset of the first symptom. The time of appearance of each symptom was placed over the line.

\section{RESULTS}

A total of 92 children were evaluated. Fifty children (54\%) were diagnosed with COVID-19 infection by a positive SARS-CoV-2 RT-PCR test. Since chronic patients may have different clinical manifestations, they were described separately from previously healthy patients.

Twenty-six patients (52\%) with confirmed COVID19 infection had a previous chronic medical condition. The most common condition was cancer $(16 \%)$, followed by chronic lung disease $(12 \%)$, obesity $(8 \%)$, chronic kidney disease $(6 \%)$ and neurological disorders $(4 \%)$. Three patients had more than one chronic condition. 
From the evaluated symptoms, 35 children presented with fever (70\%), 36\% excessive crying and dry cough; and $4 \%$ had hyposmia (Table I). Digestive symptoms were common; $24 \%$ of the patients presented only gastrointestinal symptoms. All patients without any respiratory or gastrointestinal symptoms were immunocompromised.

From the evaluated signs, 30 children presented pharyngeal erythema (60\%), 24 with irritability (24\%) and 10 with rhinorrhea and conjunctival hyperemia $(20 \%)$ (Table I). The first symptom to appear was fever in 36\% and cough in $12 \%$, followed by fatigue, rhinitis, and excessive crying each in $8 \%$ of the patients. Manifestations intentionally sought but not found in any patient were expectoration, mucopurulent rhinorrhea, posterior nasal discharge, mucopurulent conjunctival discharge, and epistaxis. Manifestations intentionally sought that were found in only one patient were nasal mucosa edema, rhonchi, cyanosis, lymphadenopathy, grunting, and wheezing.

Analyzing the diagrams of the symptom appearance chronology, we defined three different patterns: Pattern A or almost asymptomatic: with only one or two symptoms; Pattern S or sudden: onset of $4 \geq$ symptoms in the first 2436 hours; and Pattern D or disperse: sequential onset of symptoms over several days. The patterns were distributed in an irregular form in both groups; nonetheless, considering only the patients with pneumonia, the $\mathrm{S}$ pattern was found in seven of nine of the chronically ill patients, in two of eight immunocompromised patients, in four of the five patients with chronic lung disease and in three of four obese patients (Table II).

The rate of admission was significantly higher in chronically ill $(61.5 \%)$ versus healthy individuals (31.7\%); however, 7 of the 27 chronically ill individuals were admitted for previous disease decompensation. Of the 8 immunocompromised patients, two developed pneumonia. Four of the five patients with obesity also developed pneumonia.

All patients with pneumonia, except one, were admitted. Only one patient developed Kawasaki-like syndrome. Two patients required mechanical ventilation: one of them was a patient with cystic fibrosis, and the other was a kidney transplant patient who later died.

\section{DISCUSSION}

The clinical suspicion of SARS-CoV-2 infection in children has been a challenge for physicians worldwide. Many case series have been published; however, most of them are retrospective and collect few clinical features. A broader description of the disease is of paramount importance for the clinical suspicion of SARS-CoV-2
Table I Signs and Symptoms among 50 Pediatric Patients with confirmed COVID-19

\begin{tabular}{|c|c|c|c|}
\hline Symptoms ${ }^{a}$ & No. $(\%)$ & Signs & No. $(\%)$ \\
\hline $\begin{array}{l}\text { Time of symptom } \\
\text { onset }^{b}\end{array}$ & $72(33-144)$ & $\begin{array}{l}\text { Pharyngeal } \\
\text { erythema }\end{array}$ & $30(60)$ \\
\hline Fever & $35(70)$ & Irritability & $12(24)$ \\
\hline Excessive crying & $18(36)$ & Rhinorrhea & $10(20)$ \\
\hline Dry Cough & $18(36)$ & $\begin{array}{l}\text { Conjunctival } \\
\text { hyperemia }\end{array}$ & $10(20)$ \\
\hline Rhinitis & $13(26)$ & Respiratory distress & $6(12)$ \\
\hline Sore throat & $12(24)$ & Dehydration & $6(12)$ \\
\hline Headache & $11(22)$ & Crackles & $4(8)$ \\
\hline Abdominal pain & $11(22)$ & Fever & $4(8)$ \\
\hline $\begin{array}{l}\text { Conjunctival } \\
\text { hyperemia }\end{array}$ & $11(22)$ & Rash & $3(6)$ \\
\hline Vomiting & $11(22)$ & $\begin{array}{l}\text { Diminished breath } \\
\text { sounds }\end{array}$ & $3(6)$ \\
\hline Diarrhea & $11(22)$ & Rhonchi & $2(4)$ \\
\hline Shortness of breath & $10(20)$ & Nasal mucosa edema & $2(4)$ \\
\hline Fatigue & $9(18)$ & Dysphonic & $2(4)$ \\
\hline Hyporexia & $9(18)$ & Hyperemia of pillars & $2(4)$ \\
\hline Myalgia & $7(14)$ & Somnolence & $2(4)$ \\
\hline Dysgeusia & $4(8)$ & Type of symptoms & \\
\hline Arthralgia & $4(8)$ & Only respiratory & $25(50)$ \\
\hline Productive cough & $4(8)$ & Only gastrointestinal & $12(24)$ \\
\hline Rash & $4(8)$ & Both & $10(20)$ \\
\hline Hoarseness & $5(5)$ & Without any of them & $3(6)$ \\
\hline
\end{tabular}

${ }^{a}$ Hyposmia and cyanosis-2 children each; excessive daytime sleepiness in 3. ${ }^{b}$ Median (IQR) time from admission to onset of first symptom.

Table II Demographic and Clinical Characteristics, of Pediatric Patients With COVID-19 in Mexico $(N=50)$

\begin{tabular}{lccr}
\hline Characteristics & $\begin{array}{c}\text { Chronic } \\
\text { medical } \\
\text { illness }(n=26)\end{array}$ & $\begin{array}{c}\text { Previously } \\
\text { healthy } \\
(n=24)\end{array}$ & Total \\
\hline Age, ${ }^{\text {a mo }}$ & $108(26-153)$ & $\begin{array}{c}18 \\
(7.25-99.5)\end{array}$ & $\begin{array}{r}(13-159) \\
\text { Boys }\end{array}$ \\
Immunosuppression & $20(76.9)$ & $15(62.5)$ & $35(70)$ \\
Pneumonia & $8(30.8)$ & $0(0)$ & $8(16)$ \\
Admitted & $8(30.8)$ & $10(41.7)$ & $18(36)$ \\
Past antibiotic treatment & $7(26.9)$ & $4(16.7)$ & $15(30)$ \\
Past symptomatic & $19(73.1)$ & $16(66.7)$ & $35(70)$ \\
$\quad$ treatment & & & \\
Clinical patterns & & & \\
Pattern A & $4(15.4)$ & $7(29.1)$ & $11(22)$ \\
Pattern S & $12(50)$ & $9(37.6)$ & $21(42)$ \\
Pattern D & $8(30.8)$ & $10(38.8)$ & $18(36)$ \\
\hline
\end{tabular}

Values in no. (\%) or ${ }^{\mathrm{a}}$ median (IQR); Clinical patterns of disease: A: almost asymptomatic; $S$ (sudden); D (disperse). 


\section{WHAT THIS STUDY ADDS?}

- The clinical spectrum of COVID-19 in children is wider than previously described.

- Digestive symptoms and other symptoms like loss of taste or sensation should raise suspicion.

infection in children. Although our study included a limited number of patients, it explored a wider clinical spectrum in a heterogeneous population of pediatric patients.

The testing capacity for SARS-CoV-2 in Mexico is limited and reserved for patients who meet the national epidemiological definition. Many children with symptoms consistent with COVID-19 in the community are not tested and consequently not diagnosed. Our inclusion criteria allowed us to analyze patients who otherwise would not have been tested. These results may serve to reconsider epidemiological definitions for children with suspected COVID-19.

It is important to highlight that this study captured data from children who were seen or managed within a tertiary health-care institution; one-half of the patients had a chronic medical condition. Consequently, the study population is likely to primarily represent individuals with the more severe end of the disease spectrum. Statistical differences between the two groups were not calculated since the research was designed as a case series description, the sample was small, and there was a wide difference in age between both groups.

Adult patients with digestive symptoms without respiratory symptoms are rare [4], while in children it seems to be more frequent. Our results suggest that SARSCoV-2 infection often presents with nonspecific signs and symptoms, and digestive symptoms, even in the absence of respiratory symptoms, should raise suspicion. Loss of taste and smell in adults has been reported in up to half of patients [5] and proposed as an important discriminatory symptom. Chemosensory dysfunction in children is seldom reported [6], but our results suggest that both hyposmia and dysgeusia are not so rare.

Pneumonia in patients with COVID-19 has been reported in up to $64.9 \%$ of children with COVID-19 using radiologic criteria [7], but it is rarely reported using clinical criteria. We found one-third of patients with pulmonary infection, exploring only clinical features. The recognition of different clinical patterns of COVID-19 may help us recognize patients with a higher risk of poor outcomes. Our results suggest that the ' $\mathrm{S}$ ' or sudden pattern is associated with pneumonia in patients with underlying chronic conditions. Additionally, as shown in young adults [8], obesity appears to be an important risk factor for poor outcomes (pulmonary involvement) in children with COVID-19.

Clinicians should recognize that the clinical spectrum of COVID-19 in children is wider than previously described and different from the adult presentation; often with nonspecific signs and symptoms, digestive symptoms should increase clinical awareness. The order of appearance of symptoms (clinical pattern) requires more investigations, as our results suggest that it could predict outcomes.

Ethics Clearance: Institutional ethics committee of Hospital Infantil de México Federico Gómez; No. HIM-AE-004-2020, dated September 14, 2020.

Contributors: EB: conceptualized the study design; EB,DC,NS,MC: recruited patients, collected demographic and clinical data. EB,DC: analyzed and interpreted the results; EB: wrote the manuscript in Spanish; NS,DC: translated the manuscript; DC,NS,MC,EB: commented on and revised the manuscript. All authors approved the final report.

Funding: None; Competing interest; None stated.

\section{REFERENCES}

1. Dirección general de epidemiología, Secretaria de Salud. Accessed September 11, 2020. Available from: https:// www.gob.mx/salud/documentos/datos-abiertos-baseshistoricas-direccion-general-de-epidemiologia

2. Zhang L, Peres TG, Silva MVF, Camargos P. What we know so far about Coronavirus Disease 2019 in children: A meta-analysis of 551 laboratory-confirmed cases. Pediatr Pulmonol. 2020;55:2115-27.

3. Liguoro I, Pilotto C, Bonanni M, et al. SARS-COV-2 infection in children and newborns: A systematic review. Eur J Pediatr. 2020;179:1029-46.

4. Pan L, Mu M, Yang P, et al. Clinical characteristics of COVID-19 patients with digestive symptoms in Hubei, China: A descriptive, cross-sectional, multicenter study. Am J Gastroenterol. 2020;115:766-73.

5. Chi H, Chiu N-C, Peng C-C, et al. One-seventh of patients with COVID-19 had olfactory and gustatory abnormalities as their initial symptoms: A systematic review and metaanalysis. Life. 2020;10:158.

6. Mak PQ, Chung KS, Wong JSC, Shek CC, Kwan MYW. Anosmia and ageusia: Not an uncommon presentation of COVID-19 infection in children and adolescents. Pediatr Infect Dis J. 2020;39:E199-200.

7. Lu X, Zhang L, Du H, et al. SARS-CoV-2 infection in children. N Engl J Med 2020; 382:1663-1665.

8. Steinberg E, Wright E, Kushner B. In young adults with COVID-19, obesity is associated with adverse outcomes. West J Emerg Med. 2020;21:752-5. 